\title{
Fracture Forces of Various CAD/CAM Manufactured Ceramic Anterior Three-Unit Bridges
}

\author{
Kang YH*, Murali S, Gaudet S and Kugel G \\ Department of Comprehensive Care, Tufts University, USA
}

*Corresponding author: Yoon H Kang, Department of Comprehensive Care, Tufts University, USA, Email: yoon.kang@tufts.edu

\section{Research Article}

Volume 5 Issue 2

Received Date: May 19, 2020

Published Date: May 29, 2020

DOI: $10.23880 /$ oajds- 16000255

\section{Abstract}

Objectives: This study evaluated the fracture forces of anterior three-unit bridges made from various ceramic CAD/CAM materials.

Materials and Methods: The investigated materials were Ivoclar Vivadent ${ }^{\circledR}$ IPS e.max $®$ CAD (EM), $3 M^{\mathrm{TM}}$ Lava $^{\mathrm{TM}}$ Esthetic Fluorescent Full-Contour Zirconia Disc (LE) and 3M Lava Plus High Translucency Zirconia Disc shaded with Lava Plus Dyeing Liquid (LP). Wall thickness of the crowns were $1.2 \mathrm{~mm}$ (EM), $0.8 \mathrm{~mm}$ (LE), or $0.5 \mathrm{~mm}$ (LP) with connector cross-sectional areas of $16 \mathrm{~mm} 2$ (EM), $12 \mathrm{~mm} 2$ (LE), or 7mm2 (LP). EM ( $\mathrm{n}=14$ ) bridges were ground using Sirona CEREC $®$ inLab® MC XL. LE and LP bridges were milled on Sirona inLab®MC X5. Force was applied to the bridge pontic using an Instron $5566 \circledast$ universal testing machine to determine fracture force. 2-sample-t-tests $(\mathrm{p}<0.05)$ were performed for all groups.

Results: Mean fracture force was significantly higher for LE (1789N) and LP (1745N) as compared to EM (1458N). There was no statistically significant difference of mean fracture forces between LE and LP. Zirconia bridges made from LE and LP with thinner wall thicknesses and lower cross-sectional connector areas compared to EM showed higher fracture forces.

Conclusion: Within the confines of this study, zirconia bridges offer higher mechanical properties and therefore allow more tissue preserving tooth preparation.

Clinical Relevance: The clinical relevance of using novel zirconia ceramic materials is tissue preserving tooth preparation and the materials' performance with its minimum design requirements in regard to fracture forces of bridges.

Keywords: Glass ceramic; CAD/CAM; Lithium disilicate; Zirconia; Occlusal force

Abbreviations: EM: Emax; LP: Lava Plus; LE: Lava Esthetic; CAD/CAM: Computer-Aided Design and ComputerAided Manufacturing; FPDs: Fixed Partial Dentures.

\section{Introduction}

As modern dentistry is transitioning toward chairside production along with Computer-Aided Design and Computer-Aided Manufacturing (CAD/CAM), a market for restorative materials that have lower failure rates, more natural-looking profiles, and minimal tooth preparation requirement is surging. CAD/CAM technology, incorporating intraoral digital scanning, prosthetic design software, in-office milling machines, and post processing sinter furnaces, all in a digital data domain, has become a big part of restorative dentistry as its influence has been steadily increasing in the past 20 years with a much higher capability to improve patient care quality [1,2]. This digital transition is further evident in dental education as one study reports CAD/ CAM technology is part of the teaching curriculum in $93 \%$ of US dental schools that participated in the digital dentistry survey study [3]. Most well-known CAD/CAM systems may be Chairside Economical Restoration of Esthetic Ceramics or CEREC system first developed in the mid 1980's and steadily improved since [4]. One of the biggest advantages of CAD/CAM dentistry is multi-visit indirect restorations procedures such as crowns and fixed partial dentures (FPDs) can turn into one-visit chairside procedures. This represents a significant paradigm shift from traditional laboratory based 'analog dentistry' to digital dentistry that replaces 


\section{Open Access Journal of Dental Sciences}

laboratory personnel's role with in office milling machines or more robust fabrication units at the offsite laboratory [5]. Recent studies have suggested that intraoral scanned models produce more precise accuracy in digital domain while fitting of prosthetics steadily improves as does the chairside milling technology [4].

One of the main issues with digitally produced prosthetics is degree of fitting precision from mismatch between digitally created dimension transformed to milled prosthetics. One study implicated the imprecision from digital domain to physical milling transformation stems from different types of ceramic blocks sintered from different micro-sized and shaped ceramic powders [6]. CAD/CAM technology offers increased material selection options for the clinicians, however, this new availability of technology offers clinicians in the position of making on the opportunity to choose more optimal materials, in terms of precision, strength and esthetics [6,7]. CAD/CAM dentistry offers various materials choices from resin composites, polycrystalline ceramics, glass-ceramics and even resin-ceramic based materials to meet the restorative dentistry requirements in terms of mechanical strength, biocompatibility and esthetics replicability $[2,8,9]$.

One limitation is that it cannot mill multilayered ceramic prosthetics capable of providing strength and superior esthetics. Two main ceramics used for CAD/CAM milling are glass ceramics, lithium disilicate and polycrystalline based zirconia blocks. In terms of strength, polycrystalline ceramics such as zirconia show highest fracture strength at 900sMPa's, while glass ceramics such as lithium disilicate show highest fracture strengths around the mid 300sMPa's [10]. Translucency is a key parameter for esthetics and it is related to the level of crystallinity and the thickness, which dictates the strength of the prosthetics. Zirconia provides superior strength but lacks in esthetics, mainly due to its lack of translucency [11]. It is a preferred ceramic in posterior prosthetics where strength over esthetics is a key clinical preference. On the other hand, due to its relatively high strength and excellent light-optical properties, lithium disilicate continues to be one of the most popular glassceramic materials used in the fabrication of dental crowns and bridges. However, where lithium disilicate falls short is in its tooth preparation design properties which demands removal of more enamel and dentin when compared to newer CAD/CAM ceramic restoration materials.

Two of the most important materials selection criteria for clinicians are: (1) strength capable of withstanding occlusal forces from cyclical loadings, and (2) esthetics compatibility to adjacent natural dentitions. In a 2017 study by Theelke, et al. [12], mechanical properties of glassceramics and novel zirconia ceramics were compared and a zirconia product was found to have greater flexural strength supporting its indication for crown and bridge restorations. Depending on the mechanical properties and restoration location (anterior/posterior), different design guidelines have been proposed by the manufacturers. Amount of abutment reduction does affect fracture strength of ceramic prosthetics and it is important for clinicians to adapt their clinical operation based on the prosthetic material types they select [13]. It has been found that the high strength of novel zirconia ceramic materials is reflected in a decreased minimum wall thickness and connector cross sectional area as compared to materials with lower strength per each material's IFU. The clinical relevance of using novel zirconia ceramic materials is tissue preserving tooth preparation and the materials' performance with its minimum design requirements in regard to fracture forces of bridges.

In this study, we aimed to measure the fracture forces of anterior three-unit bridges made from various ceramic CAD/ CAM materials according to design recommendations.

Materials tested and compared are all monolithic ceramics:

a) Lithium disilicate,

b) Zirconia with improved translucency with UV fluorescence and

c) Zirconia chemically treated to 'layer' additional shading chemicals as a part of post milling process.

Since each material has different strength properties, 3-unit bridge preparations required different minimum crown and connector thicknesses per group based on the design recommendations. From measuring and comparing forces required to fracture these specifically dimensioned 3-unit bridges, our goal was to provide practical clinical guidance to the dental clinicians on how to choose the right CAD/CAM ceramic material for their cases to achieve (1) required prosthetics strength, (2) maximum esthetics, and (3) optimal minimum amount of tooth reduction.

\section{Materials and Methods}

Anterior 3-unit bridges are manufactured by CAD/CAM workflows to fit on a standardized fracture force test setup as shown in Figure 1. The ceramic materials to be tested are (a) lithium disilicate - Ivoclar Vivadent ${ }^{\circledR}$ IPS e.max ${ }^{\circledR}$ CAD (Ivoclar Vivadent Inc., Amherst, NY) (EM), (b) zirconia with improved translucency with UV fluorescence-3MTM LavaTM Esthetic Fluorescent Full-Contour Zirconia Disc (3M, St. Paul, MN) (LE), and (c) zirconia chemically treated to 'layer' additional shading chemicals as a part of post milling process-3MTM LavaTM Plus High Translucency Zirconia Disc shaded with Lava Plus Dyeing Liquid (3M, St. Paul, MN) (LP). Wall thickness of the crowns were $1.2 \mathrm{~mm}$ (EM), $0.8 \mathrm{~mm}$ (LE), or $0.5 \mathrm{~mm}$ (LP) with connector cross-sectional areas of $16 \mathrm{~mm} 2(\mathrm{EM}), 12 \mathrm{~mm} 2$ (LE), or $7 \mathrm{~mm} 2$ (LP) as shown 
in Figure 2. EM $(n=14)$ bridges were ground using Sirona CEREC® inLab® MC XL (Dentsply Sirona, Charlotte, NC). LE $(n=15)$ and $\operatorname{LP}(n=12)$ bridges were milled on Sirona inLabß MC X5 (Dentsply Sirona, Charlotte, NC). All restorations were heat-treated based on the manufacturers' instructions using an Ivoclar Vivadent Programat ${ }^{\circ}$ EP5000G2 furnace for crystallization (Ivoclar Vivadent Inc., Amherst, NY) (EM) or 3M Lava Furnace 200 for final sintering (3M, St. Paul, MN)
(LE, LP). Complete summary of sample descriptions are presented in Table 1. All restorations were cemented on tooth shaped abutments made from a composite material to simulate dentin. Force was applied to the bridge pontic using an Instron $5566 \AA$ universal testing machine to determine fracture force. 2 -tailed-t-tests $(\mathrm{p}<0.05)$ have been performed for all groups.

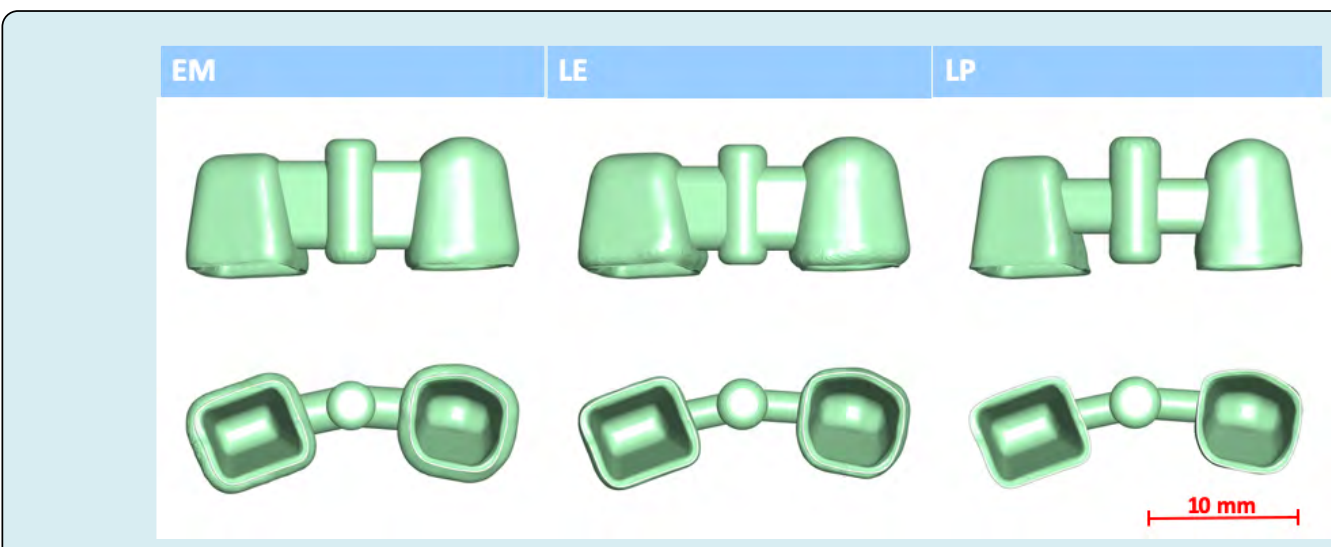

Figure 1: CAD/CAM designed anterior 3-unit bridge for a standardized fracture force test setup.

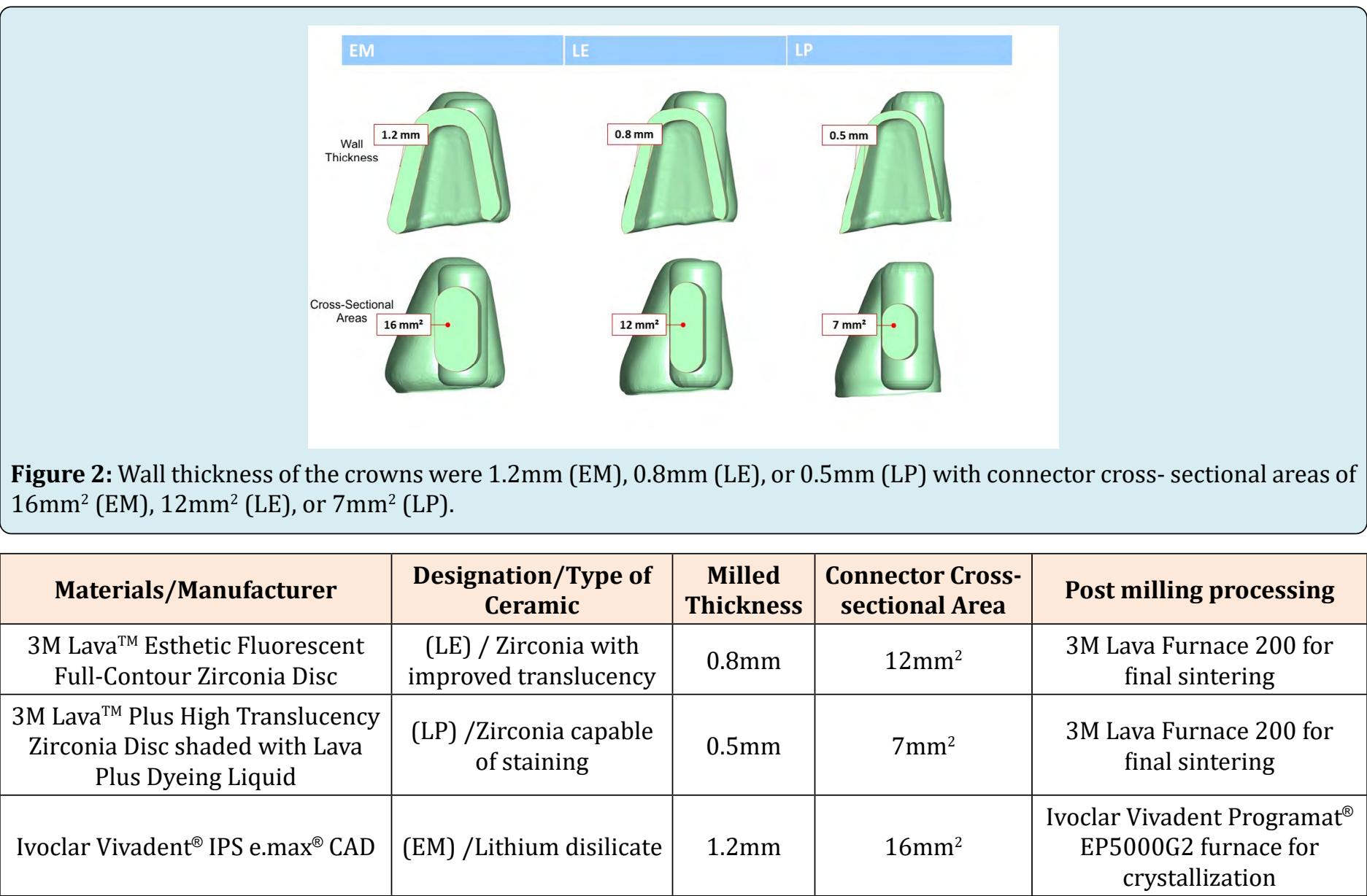

Table 1: Tested materials with fabricated thickness dimensions and post milling processing method. 


\section{Results}

Mechanical testing results are presented in Table 2 and Figure 3. Mean fracture forces measured were $1789 \mathrm{~N}$ for LE and $1745 \mathrm{~N}$ for LP and $1458 \mathrm{~N}$ for EM as shown in Table 2. The differences in fracture forces of EM versus LE and EM versus LP were both found to have $\mathrm{p}<0.05$ as shown in Figure 3. When compared, there was no statistically significant difference of mean fracture forces between LE and LP.

\begin{tabular}{|c|c|c|c|}
\hline Material & Mean Fracture Force (N) & Standard Deviation (N) & Statistical groups \\
\hline EM & 1458 & 128 & $\mathrm{a}$ \\
\hline LE & 1745 & 288 & $\mathrm{~b}$ \\
\hline LP & 1789 & 201 & $\mathrm{~b}$ \\
\hline
\end{tabular}

$\mathrm{p}<0.05 \mathrm{a}$ vs $\mathrm{b}$.

Table 2: Mean fracture forces for EM, LE and LP when force as applied to the bridge pontic.

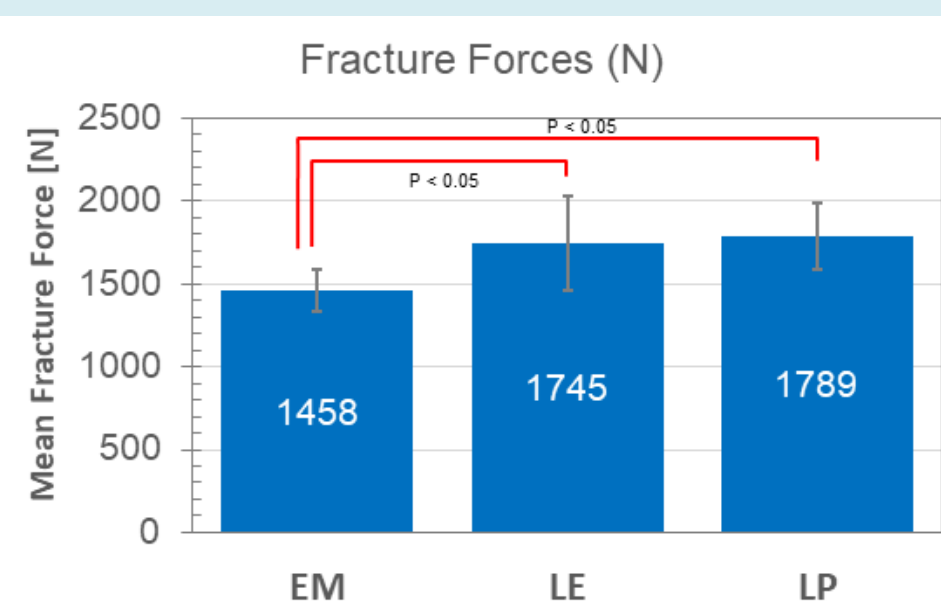

Figure 3: Mean fracture forces for EM, LE, and LP when force was applied to the bridge pontic.

\section{Discussion \& Conclusion}

Zirconia bridges made from LE and LP blocks with thinner wall thicknesses and smaller cross-sectional connector areas Figure 2 compared to the samples from EM blocks still yielded higher fracture forces. Instead of comparing the strength of the ceramics, samples were fabricated to the respective design recommendation of the ceramics, which is more direct indicator of mechanical performance under the force generated from occlusion. In terms of strength, polycrystalline ceramics such as zirconia have highest fracture strengths at $900 \mathrm{~s} \mathrm{MPa}$, followed by another type of polycrystalline ceramic such as aluminum oxide with fracture strengths in the mid 500s MPa and glass ceramics such as lithium disilicate at around the mid 300s MPa [10]. When selecting stronger ceramics, clinicians have the advantage of being able to preserve more healthy dentin structures with thinner prosthetics. In addition to being biologically conservative, thinner prosthetics offer an advantage of 'fitting' the new prosthetics into the existing occlusion of the patients with more clinical flexibility, resulting benefits such as better countering of cyclical stress from parafunctional mechanisms. However, stronger ceramic with thin wall thickness means possible compromise of esthetics. Two types of ceramics used in this study, lithium disilicate and zirconia represent the opposite of this strength-esthetics spectrum. Better esthetics with lower strength for lithium disilicate and poor esthetics with higher strength for zirconia. When selecting ceramics in terms of strength, prosthetics must be able to withstand well beyond the potential maximum forces generated from occlusion. Reported values of forces exerted to dentitions range from $100 \mathrm{~N}$ to $635 \mathrm{~N}$ ranging from light chewing and swallowing motions to hard bite actions [1416].

As reported from this study, all three ceramics, prepared to optimal thickness, showed that fabricated prosthetics will fail at values well exceeding $635 \mathrm{~N}$ (1458-1798 N). Another important clinical decision factor is the quality of esthetics from the selected ceramic. Translucency is related to ceramic thickness which is directly related to the strength 
of the prosthetics. Esthetics quality from translucency of lithium disilicate is superior to that of zirconia, and selection between lithium disilicate and zirconia is in essence balances the tradeoffs among esthetics, mechanical performance, and biological conservation [17-20]. CAD/CAM technology has a limitation of only being able to produce monolithic based crowns or FPDs. As a result, quality of esthetics mainly depends upon the ceramic material chosen. Innovations with materials processing ventures to overcome this and to achieve both high mechanical performance with improved esthetics. This is addressed in one approach by $3 \mathrm{M}$ Lava Esthetic which offers a translucency comparable to e.max CAD, a pre-shaded multilayer concept in all Vita Classic Shades including inherent fluorescence and sufficient mechanical performance to enable up to 3-unit restoration in posterior and anterior region.

Another example is 3M LavaTM Plus High Translucency Zirconia which clinicians can add shade after the milling process to achieve desired esthetics. This study evaluated the mechanical property of the three selected monolithic ceramics featuring different esthetics properties. They offer options on biological reduction amount and different degree of esthetics achieved by the manufactured prosthetics. These options, when carefully considered based on the individual clinical case by cases, will bolster clinicians significantly to successfully complete cases that will restore stable and functional occlusion and satisfactory esthetics results. Table 3 summarized each ceramics' advantages and disadvantages based on this study's findings. In depth understanding of CAD/CAM based ceramic blocks by practicing clinicians should be one of the key elements for selecting the most optimal restoration material leading to successful completion of restoration cases.

\section{References}

1. Miyazaki T, Hotta Y, Kunii J, Kuriyama S, Tamaki Y (2009) A review of dental CAD/CAM: current status and future perspectives from 20 years of experience. Dent Mater J 28(1): 44-56.

2. Blatz MB, Conejo J (2019) The Current State of Chairside Digital Dentistry and Materials. Dent Clin North Am 63(2): 175-197.

3. Prager MC, Liss H (2020) Assessment of Digital Workflow in Predoctoral Education and Patient Care in North American Dental Schools. J Dent Educ 84(3): 350-357.

4. Zaruba M, Mehl A (2017) Chairside systems: a current review. Int J Comput Dent 20(2): 123-149.

5. Patel N (2014) Contemporary dental CAD/CAM: modern chairside/lab applications and the future of computerized dentistry. Compend Contin Educ Dent 35(10): 739-746.

6. Piras FF, Ferruzzi F, Ferrairo BM, Ramalho IS, Bonfante EA, et al. (2020) Analysis of correlation between optical and microtomography measurements of cementation space in CAD-CAM ceramic crowns. J Prosthet Dent.

7. Nasr E, Makhlouf AC, Zebouni E, Makzoume J (2019) Allceramic Computer-aided Design and Computer-aided Manufacturing Restorations: Evolution of Structures and Criteria for Clinical Application. J Contemp Dent Pract 20(4): 516-523.

8. Li RW, Chow TW, Matinlinna JP (2014) Ceramic dental biomaterials and CAD/CAM technology: state of the art. J Prosthodont Res 58(4): 208-216.

9. Spitznagel FA, Boldt J, Gierthmuehlen PC (2018) CAD/ CAM Ceramic Restorative Materials for Natural Teeth. J Dent Res 97(10): 1082-1091.

10. Deng Y, Lawn BR, Lloyd IK (2002) Characterization of damage modes in dental ceramic bilayer structures. J Biomed Mater Res 63(2): 137-145.

11. Zhang Y, Lawn BR (2018) Novel Zirconia Materials in Dentistry. J Dent Res 97(2): 140-147.

12. Theelke B (2017) Flexural Strength of a Novel Esthetic Full-Contour Zirconia Compared to Other Ceramics. in 017 IADR/AADR/CADR General Session (San Francisco, California). 2017. San Francisco, California.

13. Falahchai M, Hemmati YB, Asli HN, Rezaei E (2020) Effect of Tooth Preparation Design on Fracture Resistance of Zirconia-Reinforced Lithium Silicate Overlays. J Prosthodont.

14. Lundgren D, Laurell L (1986) Occlusal force pattern during chewing and biting in dentitions restored with fixed bridges of cross-arch extension. I. Bilateral end abutments. J Oral Rehabil 13(1): 57-71.

15. Proeschel PA, Morneburg T (2002) Task-dependence of activity/ bite-force relations and its impact on estimation of chewing force from EMG. J Dent Res 81(7): 464-468.

16. Umesh S, Padma S, Asokan S, Srinivas T (2016) Fiber Bragg Grating based bite force measurement. J Biomech 49(13): 2877-2881.

17. Harada K, Raigrodski AJ, Chung KH, Flinn BD, Dogan S, et al. (2016) A comparative evaluation of the translucency of zirconias and lithium disilicate for monolithic restorations. J Prosthet Dent 116(2): 257-263. 
Open Access Journal of Dental Sciences

18. Zarone F, Mauro MID, Ausiello P, Ruggiero G, Sorrentino R (2019) Current status on lithium disilicate and zirconia: a narrative review. BMC Oral Health 19(1): 134.

19. Zhang F, Reveron H, Spies BC, Meerbeek BV, Chevalier J (2019) Trade-off between fracture resistance and translucency of zirconia and lithium-disilicate glass ceramics for monolithic restorations. Acta Biomater 91: 24-34.

20. Fonzar RF, Carrabba M, Sedda M, Ferrari M, Goracci C (2017) Flexural resistance of heat-pressed and CADCAM lithium disilicate with different translucencies. Dent Mater 33(1): 63-70. 